\title{
Praxisrelevante Informationen auf den Punkt gebracht
}

Über den heutigen Stand der Intraoralscanner-Technik informiert die $3 \mathrm{M}$ Health Care Academy in unterschiedlichen Fortbildungen. Wer allgemeine Informationen zum Thema digitale Abformung wünscht, für den empfiehlt sich der Kurs „Discover the next generation: Intraoralscanner im Fokus von Wissen-

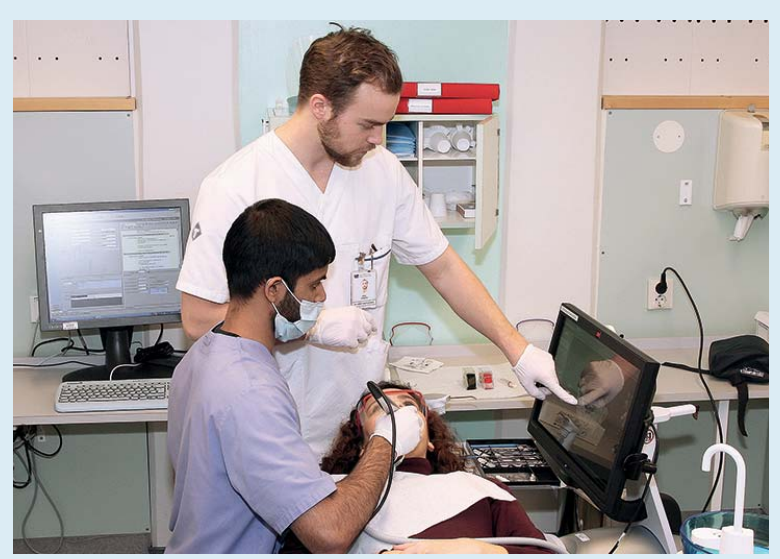

schaft und Praxis“. Hier referiert ein Wissenschaftler über Indikationen und Anwendungsbereiche unterschiedlicher Intraoralscanner und erläutert, für wen sich der Einstieg in die digitale Abformung lohnt. Anschließend widmet sich ein Anwender des 3M True Definition Scanner konkreten Fragestellungen rund um die Integration von Intraoralscannern in den Praxisalltag. Es folgen eine Live-Demonstration am Modell sowie die Gelegenheit, den 3M True Definition Scanner selbst zu testen.

Für Zahnärzte, die sich über die Anforderungen an Praxis und Labor bei Einsatz des Scanners informieren möchten, eignet sich der Kurs „Hand in
Hand zu passgenauer Prothetik - Digitale Abformung als Bindeglied zwischen Praxis und Labor“. Hier werden Zahnarzt und Zahntechniker als Team über alle relevanten Details rund um die Scannerintegration, -anwendung und die Weiterverarbeitung der Daten aufgeklärt. Zum Abschluss werden eine Live-Demonstration am Patienten sowie ein Workshop geboten. Bei Interesse lassen sich die erworbenen Kenntnisse im Rahmen einer Praxis-Hospitation vertiefen.

Eine Terminübersicht sowie Detailinformationen zu allen Kursen bietet ein Programmheft, das über die Hotline 0800 / 2753773 kostenfrei bestellbar ist und unter www.3MESPE.de in der Rubrik Veranstaltungen zum Download bereitsteht.

Nach einer Pressemitteilung der

3M Deutschland GmbH, Seefeld 